\title{
Multiclass Image Labeling with Semidefinite Programming
}

\author{
Jens Keuchel \\ Institute of Computational Science, ETH Zurich, 8092 Zurich, Switzerland \\ Jens.Keuchel@inf .ethz.ch
}

\begin{abstract}
We propose a semidefinite relaxation technique for multiclass image labeling problems. In this context, we consider labeling as a special case of supervised classification with a predefined number of classes and known but arbitrary dissimilarities between each image element and each class. Using Markov random fields to model pairwise relationships, this leads to a global energy minimization problem. In order to handle its combinatorial complexity, we apply Lagrangian relaxation to derive a semidefinite program, which has several advantageous properties over alternative methods like graph cuts. In particular, there are no restrictions concerning the form of the pairwise interactions, which e.g. allows us to incorporate a basic shape concept into the energy function. Based on the solution matrix of our convex relaxation, a suboptimal solution of the original labeling problem can be easily computed. Statistical groundtruth experiments and several examples of multiclass image labeling and restoration problems show that high quality solutions are obtained with this technique.
\end{abstract}

\section{Introduction}

Classification of extracted image elements (e.g. pixels, patches, objects) or in short image labeling is a fundamental issue in computer vision. Based on a predefined number of classes, the goal is to assign each image element to one of these classes according to some suitable criterion. Not considering the corresponding problem of learning adequate class representations here, we assume that fixed dissimilarity values between each image element in comparison to the different classes are given in advance. Depending on the application, these dissimilarities may be based on previously known class prototypes, resulting in an image restoration problem (the image features are noisy measurements of the given prototypes), or on class representations that can be estimated from training data (by making certain assumptions about the distribution of the image features).

As illustrating examples consider the images given in Figure 1 based on the observed values, we want to either restore the original image by determining the 'true' intensity for each pixel, or classify the pixels according to some measured feature (like texture). To this end, a compromise between two competing forces is usually sought [1]: on the one hand, we look for classifications that best conform to the observed feature values, while on the other hand - assuming that natural 

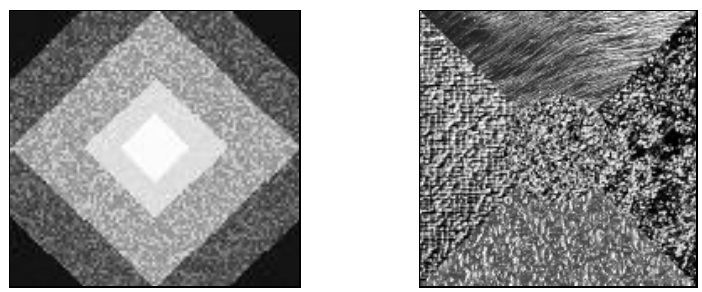

Fig. 1. Multiclass image restoration/labeling. Restore a gray-value image from noisy measurements (left). Label image parts according to their texture (right).

images are mostly smooth, except for occasional region boundaries — spatially neighboring pixels should receive similar labels.

In order to find a labeling which captures this trade-off, we seek to minimize a global energy function which involves pairwise relationships among the objects. This kind of problem has a long history in the literature, in particular as it arises naturally from the well-studied theory of Markov random fields, a statistical framework that builds the core of many image processing applications [1, 2, 3, 4,

Using integer variables $x_{i}$ to indicate the label for each image element $i$, the resulting problem can be formulated as minimizing an energy functional of the following general form [5]:

$$
E(x)=\sum_{i} C_{i}\left(x_{i}\right)+\sum_{\langle i, j\rangle} V_{i j}\left(x_{i}, x_{j}\right),
$$

where the second term sums over all pairwise interacting image elements. The energy (11) comprises two terms familiar from many regularization approaches: a data-fitting term and a smoothness term modeling spatial context. In more detail, the data-fitting term measures the dissimilarity $C_{i}\left(x_{i}\right)$ between element $i$ and class $x_{i}$ (assignment costs), while the smoothness term evaluates the disagreement $V_{i j}\left(x_{i}, x_{j}\right)$ of the labels for related pairs $i, j$ (separation costs).

Due to the integer constraint on the variables $x_{i}$ the optimization problems obtained from (11) are much more difficult than standard regularization problems. In fact, apart from a few special cases, they are in general NP-hard [5]. Accordingly, different methods have been proposed to find good minimizers of (special instances of) the energy (1) efficiently, like the ICM-algorithm [3, the graduated non-convexity approach [6], different versions of annealing procedures [2, 7], local search heuristics based on graph cuts [5], or linear programming relaxations [8].

Recently, a semidefinite relaxation approach was presented to approximate the minimal solution of the energy (1) for the special case of binary labeling problems 9]. At the cost of an increased but still moderate computational complexity, the tightness of this relaxation method results in high quality combinatorial solutions. This fact along with the recent success of semidefinite relaxation for other combinatorial optimization problems [10, 11, 12] motivated us to extend the approach from 9 to image labeling problems involving multiple classes. In contrast to other methods $[5,8,13$ the resulting approximation technique needs 
no special assumptions with respect to the pairwise interactions between variables given by $V_{i j}\left(x_{i}, x_{j}\right)$ in (1), so that it is applicable to a very general class of problems. In particular, each pair of image elements can have its own distinct, arbitrary interaction function, which will allow us to include basic 'shape' information into the labeling problem (see Section 44). Although recent efforts show that e.g. graph cut methods can also be adapted to less restricted problems [14,15, they are not able to deal with the energy (11) in the general case.

Other favorable properties of our approach include: As it yields a convex semidefinite program (SDP), the global optimum of the relaxation can be computed to arbitrary precision in polynomial time, without the need to heuristically optimize any tuning parameters (cf. [16]). Like the LP relaxation presented in [8], we obtain probabilities for each point-label pair, which allows us to define sophisticated rounding schemes. Moreover, the SDP relaxation gives a lower bound on the optimal objective value that can be used to estimate the per-instance approximation error.

\section{Problem Formulation}

In order to apply semidefinite relaxation to the image labeling problem, we first derive a trace formulation of the energy functional (11). To this end, let $k$ denote the number of possible classes present in the image. Furthermore, we indicate the class membership of each image element $i$ by a vector $x_{i} \in\left\{e_{1}, \ldots, e_{k}\right\}$ taking as value one of the $k$ standard unit vectors from $\mathbb{R}^{k}$. Assuming that the image contains $n$ elements, the indicator vectors form the rows of the labeling matrix $X \in \mathbb{R}^{n \times k}$.

Using these definitions, and denoting the trace of a matrix $A$ by $\operatorname{Tr}(A)=$ $\sum_{i} A_{i i}$, we obtain the following formulation of the energy functional (11):

$$
\begin{aligned}
E(x) & =\sum_{i=1}^{n} \sum_{a=1}^{k} C_{i}(a) x_{i a}+\sum_{\langle i, j\rangle} \sum_{a=1}^{k} \sum_{b=1}^{k} V_{i j}(a, b) x_{i a} x_{j b} \\
& =\operatorname{Tr}\left(C X^{\top}+\sum_{a, b} V(a, b) X I^{a b} X^{\top}\right)
\end{aligned}
$$

where $C \in \mathbb{R}^{n \times k}$ contains the costs $C_{i a}=C_{i}(a)$ for assigning object $i$ to class $a$, $V(a, b) \in \mathbb{R}^{n \times n}$ comprises the separation costs of related objects for a fixed label pair $(a, b)$, and $I^{a b} \in \mathbb{R}^{k \times k}$ is the matrix with the only non-zero entry $I_{a b}^{a b}=1$.

For many image labeling problems, the separation costs in (1) can be decomposed into two factors:

$$
V_{i j}\left(x_{i}, x_{j}\right)=P_{i j} D\left(x_{i}, x_{j}\right),
$$

where the weight $P_{i j}$ indicates the strength of the relation between image elements $i, j$, and $D\left(x_{i}, x_{j}\right)$ measures the distance between the two labels $x_{i}, x_{j}$. In this case, the trace formulation of the energy functional (2) simplifies to:

$$
E(x)=\operatorname{Tr}\left(C X^{\top}+P X D X^{\top}\right),
$$


with matrices $P \in \mathbb{R}^{n \times n}$ and $D \in \mathbb{R}^{k \times k}$. A typical example is the discontinuity preserving model of metric Potts interaction penalties [5], which are defined as

$$
P_{i j} D\left(x_{i}, x_{j}\right)=\lambda\left\|x_{i}-x_{j}\right\|^{2}=2 \lambda\left(1-x_{i}^{\top} x_{j}\right)
$$

for associated (neighboring) image elements $i, j$. Controlling the desired smoothness by the fixed parameter $\lambda \in \mathbb{R}^{+}$, seperation costs of this form encourage image regions with constant labels without penalizing sharp boundaries too much. As can be verified easily, in terms of the trace formulation (4) the Potts interactions are expressed by symmetric matrices $P$ with non-zero entries $P_{i j}=\lambda$ for associated objects $i, j$, and $D=E-I$, where $E$ and $I$ are the matrix of all ones and the identity matrix, respectively.

However, in contrast to other image labeling methods [5,8, our semidefinite relaxation approach will not require any specific form of the separation costs; in fact, they are allowed to be non-symmetric or may vary depending on the labels for a fixed object pair. Putting everything together, we consider the following optimization problem for multiclass image labeling:

$$
\begin{aligned}
z^{*}:=\min _{X \in \mathbb{R}^{n \times k}} & \operatorname{Tr}\left(C X^{\top}+\sum_{a, b} V(a, b) X I^{a b} X^{\top}\right) \\
\text { s.t. } & X e^{k}=e^{n} \\
& X_{i a} \in\{0,1\} \quad \forall 1 \leq i \leq n, 1 \leq a \leq k,
\end{aligned}
$$

where $e^{j} \in \mathbb{R}^{j}$ denotes the vector of all ones of appropriate size. The first constraint in (6) requires each row of $X$ to sum to one, which in connection with the second constraint ensures that each row corresponds to a unit vector $e_{i} \in \mathbb{R}^{k}$.

This combinatorial optimization problem resembles the generalized quadratic assignment problem (QAP, see, e.g., [17]), which has the objective to optimally place $n$ given activities at $n$ given locations by minimizing a cost function of the form $\operatorname{Tr}\left(A X B X^{\top}-2 C X^{\top}\right)$ over the set of permutation matrices $X \in \mathbb{R}^{n \times n}$ (with $A, B, C \in \mathbb{R}^{n \times n}$ ). In fact, we can interpret (6) as an uncapacitated version of a general QAP where multiple activities are allowed to be placed at the same location [8. In the context of solving the NP-hard QAP, semidefinite relaxation approaches have attracted considerable interest [10,18. In the next section, we will show how these methods can be generalized to also find approximate solutions for our less restricted labeling problem (6).

\section{Semidefinite Relaxation}

Following the QAP relaxation presented in [10, we apply Lagrangian relaxation to the image labeling problem (6). For ease of notation, we use the simplified form (4) of the energy here; the result for the more general case (2) is derived analogously and will be stated later. As a first step, we represent the constraints in (6) in quadratic form, which results in the following equivalent problem: 


$$
\begin{aligned}
z^{*}=\min _{X \in \mathbb{R}^{n \times k}} & \operatorname{Tr}\left(P X D X^{\top}+C X^{\top}\right) \\
\text { s.t. } & \left\|X e^{k}-e^{n}\right\|^{2}=0 \\
& X_{i a}^{2}-X_{i a}=0 \quad \forall i, a .
\end{aligned}
$$

Using the Lagrange multipliers $W \in \mathbb{R}^{n \times k}$ and $u_{0} \in \mathbb{R}$, we add the constraints to the objective function, and perform relaxation by virtue of the "minimax inequality" [12]:

$$
\begin{gathered}
z^{*}=\min _{X} \max _{W, u_{0}} \operatorname{Tr}\left(P X D X^{\top}+C X^{\top}\right)+\sum_{i, a} W_{i a}\left(X_{i a}^{2}-X_{i a}\right) \\
+u_{0}\left(X e^{k}-e^{n}\right)^{\top}\left(X e^{k}-e^{n}\right) \\
\geq \max _{W, u_{0}} \min _{X} \operatorname{Tr}\left(P X D X^{\top}+C X^{\top}\right)+\operatorname{Tr}\left(W(X \circ X-X)^{\top}\right) \\
+u_{0} \operatorname{Tr}\left(X E_{k} X^{\top}-2 E_{n \times k} X^{\top}\right)+u_{0} n \\
=\max _{W, u_{0}} \min _{X} \operatorname{Tr}\left(P X D X^{\top}+W(X \circ X)^{\top}+X\left(u_{0} E_{k}\right) X^{\top}\right. \\
\left.+\left(C-W-2 u_{0} E_{n \times k}\right) X^{\top}\right)+u_{0} n .
\end{gathered}
$$

Here, $X \circ X$ denotes the Hadamard (elementwise) product of two matrices, and $E_{k}$ and $E_{n \times k}$ are matrices of all ones of appropriate dimension.

Next we homogenize the objective function by multiplying $X$ with a constrained scalar $x_{0}= \pm 1$, which increases the dimension of the problem by one. The additional constraint is then inserted into the objective function by introducing the Lagrange multiplier $w_{0} \in \mathbb{R}$ :

$$
\begin{aligned}
z^{*} \geq \max _{W, u_{0}} \min _{X, x_{0}^{2}=1} \operatorname{Tr}\left(P X D X^{\top}+W(X \circ X)^{\top}+X\left(u_{0} E_{k}\right) X^{\top}\right. \\
\left.+x_{0}\left(C-W-2 u_{0} E_{n \times k}\right) X^{\top}\right)+u_{0} n x_{0}^{2} \\
\geq \max _{W, u_{0}, w_{0}} \min _{X, x_{0}} \begin{array}{l}
\operatorname{Tr}\left(P X D X^{\top}+W(X \circ X)^{\top}+X\left(u_{0} E_{k}\right) X^{\top}\right. \\
+
\end{array} \\
\left.+x_{0}\left(C-W-2 u_{0} E_{n \times k}\right) X^{\top}\right)+u_{0} n x_{0}^{2}+w_{0} x_{0}^{2}-w_{0}=: s_{d}^{*} .
\end{aligned}
$$

Transforming the problem variables $x_{0}$ and $X$ into a vector by defining $y:=$ $\left(\begin{array}{c}x_{0} \\ \operatorname{vec}(X)\end{array}\right)$, we obtain

$$
s_{d}^{*}=\max _{W, u_{0}, w_{0}} \min _{y} y^{\top}\left(L_{P, D, C}+A_{W, w_{0}}+u_{0} F\right) y-w_{0},
$$

with

$$
\begin{aligned}
& L_{P, D, C}:=\left(\begin{array}{cc}
0 & \frac{1}{2} \operatorname{vec}(C)^{\top} \\
\frac{1}{2} \operatorname{vec}(C) & D \otimes P
\end{array}\right), \\
& A_{W, w_{0}}:=\left(\begin{array}{cc}
w_{0} & -\frac{1}{2} \operatorname{vec}(W)^{\top} \\
-\frac{1}{2} \operatorname{vec}(W) & \operatorname{Diag}(\operatorname{vec}(W))
\end{array}\right) \text {, } \\
& F:=\left(\begin{array}{cc}
n & -\left(e^{n k}\right)^{\top} \\
-e^{n k} & E_{k} \otimes I
\end{array}\right)
\end{aligned}
$$


Here, $\operatorname{vec}(X)$ is the vector containing the stacked columns $x_{i}$ of $X, \operatorname{Diag}(w)$ is the diagonal matrix formed from the vector $w$, and $A \otimes B$ denotes the Kronecker product of two matrices.

There is a hidden semidefinite constraint in (8): the inner minimization is bounded below only if the matrix in the quadratic term is positive semidefinite, in which case the corresponding minimum becomes zero (cf. [10]). Indicating positive semidefiniteness of a matrix by $X \succeq 0$, this finally yields the following relaxation of (6):

$$
\begin{aligned}
s_{d}^{*}=\max _{W, u_{0}, w_{0}} & -w_{0} \\
\text { s.t. } & L_{P, D, C}+A_{W, w_{0}}+u_{0} F \succeq 0 .
\end{aligned}
$$

To obtain a direct semidefinite relaxation of (6) , we derive the Lagrangian dual of (12). To this end, first observe that the matrix in (10) can be split into $A_{W, w_{0}}=\sum_{i=0}^{n k} w_{i} A_{i}$ by defining $w:=\operatorname{vec}(W)$ and sparse symmetric, $n k+$ 1-dimensional matrices $A_{i}$ with the only non-zero entries $\left(A_{i}\right)_{i+1, i+1}=1$ and (for $i \neq 0)\left(A_{i}\right)_{1, i+1}=\left(A_{i}\right)_{i+1,1}=-\frac{1}{2}$. Using the dual positive semidefinite matrix variable $Y \in \mathbb{R}^{n k+1 \times n k+1}$, we get

$$
\begin{gathered}
s_{d}^{*}=\max _{w_{0}, w, u_{0}} \min _{Y \succeq 0}-w_{0}+\operatorname{Tr}\left(Y\left(L_{P, D, C}+\sum_{i=0}^{n k} w_{i} A_{i}+u_{0} F\right)\right) \\
\leq \min _{Y \succeq 0} \max _{w_{0}, w, u_{0}} \operatorname{Tr}\left(L_{P, D, C} Y\right)+w_{0}\left(\operatorname{Tr}\left(A_{0} Y\right)-1\right)+\sum_{i=1}^{n k} w_{i} \operatorname{Tr}\left(A_{i} Y\right) \\
+u_{0} \operatorname{Tr}(F Y) \quad=: s_{p}^{*} .
\end{gathered}
$$

As the inner maximization is unconstrained, this minimization problem is finite valued only if all the factors in the last three terms are zero. Using this hidden constraint, we finally obtain the following semidefinite program (SDP) as the dual of (12):

$$
\begin{aligned}
s_{p}^{*}=\min _{Y \succeq 0} & \operatorname{Tr}\left(L_{P, D, C} Y\right) \\
\text { s.t. } & \operatorname{Tr}\left(A_{0} Y\right)=1 \\
& \operatorname{Tr}\left(A_{i} Y\right)=0 \quad \forall 1 \leq i \leq n k \\
& \operatorname{Tr}(F Y)=0 .
\end{aligned}
$$

The connection of this semidefinite relaxation with the original integer problem (6) now becomes obvious: the binary labeling matrix $X \in \mathbb{R}^{n \times k}$ is first transformed into a vector $\operatorname{vec}(X)$ and then lifted into the higher, $(n k+1)^{2}$ dimensional space of positive semidefinite matrices by setting

$$
Y:=\left(\begin{array}{c}
1 \\
\operatorname{vec}(X)
\end{array}\right)\left(1, \operatorname{vec}(X)^{\top}\right) .
$$

The relaxation consists in discarding the intractable rank one constraint on this matrix $Y$, and minimizing over the more general space of $n k+1$-dimensional, 
positive semidefinite matrices instead (cf. [9]). Besides the $A_{0}$-constraint, which is an artificial one to enable the homogenization of the objective function, the other constraints in (13) directly correspond to the constraints in the original problem formulation (6): the $A_{i}$-constraints guarantee that the diagonal and the first row (and column) of $Y$ are identical, thus modeling the $\{0,1\}$-constraint on the entries of $X$, whereas the $F$-constraint is derived from the sum-one-constraint on the indicator vectors constituting the rows of $X$.

Regarding the more general case of the energy functional (2), the only difference during the relaxation process occurs in the derivation of (8) : instead of the matrix $L_{P, D, C}$ from (9), the term $\sum_{a, b} V(a, b) X I^{a b} X^{\top}$ in (2) yields the matrix

$$
L_{V, C}:=\left(\begin{array}{cc}
0 & \frac{1}{2} \operatorname{vec}(C)^{\top} \\
\frac{1}{2} \operatorname{vec}(C) & \sum_{a, b} I^{a b} \otimes V(a, b)
\end{array}\right)=\left(\begin{array}{cc}
0 & \frac{1}{2} \operatorname{vec}(C)^{\top} \\
\frac{1}{2} \operatorname{vec}(C) & V
\end{array}\right),
$$

with $V \in \mathbb{R}^{n k \times n k}$ being composed blockwisely of the $V(a, b)$-matrices. Hence, we obtain the corresponding general semidefinite relaxation of (6) by simply replacing $L_{P, D, C}$ with $L_{V, C}$ in (12) and (13).

Concerning the solvability of the SDP relaxation (13), we have the following lemma (cf. [10]):

Lemma 1. A feasible solution matrix $Y$ for (13) is singular, with at least $n$ of its eigenvalues being equal to zero.

Proof. The constraint matrix $F \neq 0$ is positive semidefinite: as can easily be calculated, its non-zero eigenvalues are $\lambda_{n k+1}=n+k$ and $\lambda_{n(k-1)+2}=\cdots=$ $\lambda_{n k}=k$. As $Y$ is also positive semidefinite, the constraint $\operatorname{Tr}(F Y)=\operatorname{Tr}(Y F)=0$ in (13) directly implies that $Y F$ has to be the null-matrix [19, Lemma 2.9]. Hence, $Y F_{i}=0$ for each column $F_{i}$, which shows the singularity of $Y$. As exactly $n$ columns $F_{i}$ of $F$ are linearly independent (namely $i=2, \ldots, n+1$ ), the dimension of the null space $\operatorname{ker}(Y)$ is at least $n$.

Lemma 1 implies that the semidefinite program (13) has no strictly interior point. On the other hand, we can always define a strictly interior point for the corresponding dual SDP (12) by setting $u_{0}=0$ and choosing $w_{0}$ and the entries of $W$ large enough to make the diagonal of the matrix $L_{P, D, C}+A_{W, w_{0}}$ as dominant as necessary to yield a positive definite matrix. Hence, the Slater condition holds for the dual, so that by the strong duality theorem for SDP [19], there is no duality gap: $s_{p}^{*}=s_{d}^{*}$ (for more details about the elegant duality theory for SDP, we refer to [16]).

Due to Lemma 1, however, it is not guaranteed that the optimal value of the dual SDP (12) is attained. Therefore, interior point methods can suffer from instability when solving the SDP relaxation and may not converge. One possibility to circumvent this problem is to project the SDP (13) onto a lower dimensional face of the semidefinite cone 10. However, as we only need the optimal solution of the primal SDP (and not the dual), we revert to a non-interior point algorithm to solve the SDP relaxation instead: the PENNON SDP solver [20] is based on a generalized version of the augmented Lagrangian method, and therefore does not need to compute the optimum of the dual SDP (12). 


\section{Experimental Results}

In this section, we experimentally investigate the performance of the SDP relaxation for one- and two-dimensional labeling problems. In this context, we need to derive a feasible integer solution of the original combinatorial problem (6) based on the solution matrix $Y^{*}$ of the primal SDP relaxation (13). Since the first column of $Y^{*}$ originally corresponds to $Y_{1}=\left(\begin{array}{c}1 \\ \operatorname{vec}(X)\end{array}\right)$ (cf. (14) ), we obtain an approximation $\tilde{Y}$ of the binary matrix $X$ by reshaping $Y_{1}$ appropriately: starting with the second entry, the columns of $\tilde{Y}$ are formed by blocks of length $n$ in $Y_{1}$. As in particular, the constraints on $Y$ in (13) ensure that each row $\tilde{y}_{i}$ of $\tilde{Y}$ sums to one, the entries can be interpreted as probabilities of assigning the different labels. This suggests to define the label of object $i$ (and thus the position of the one-entry in the corresponding class indicator vector $x_{i}$ ) by simply seeking the largest value in $\tilde{y}_{i}$. However, the difference $\Delta \tilde{y}_{i}$ between the two highest probability values in $\tilde{y}_{i}$ may be quite small for some objects. To take such 'doubtful labelings' into account, we follow a slightly different idea to obtain the final solution: In a first step, the labeling is fixed to the most likely class only for those objects $i$ where the difference $\Delta \tilde{y}_{i}$ is bigger than a threshold $\Delta_{\min }$. In subsequent steps, the current labeling and the remaining probabilities $\tilde{y}_{i}$ are combined into the matrix $\tilde{X}$, and modified contributions $\tilde{z}_{i}^{\top}=2 P_{i} \tilde{X} D+C_{i}$ to the objective value are calculated for the remaining objects. Seeking the smallest value $\tilde{z}_{j a}$ within all vectors $\tilde{z}_{i}$, we then fix the label for the corresponding object $j$ to $a$. In this way, the strong labels are taken into account when setting the doubtful labelings according to the objective function.

Statistical Ground Truth. As a first experiment, we measure the performance of the multiclass SDP relaxation (13) statistically. To this end, we perform the following binary $(k=2)$ ground-truth experiment (cf. 9]): A synthetic onedimensional signal (Figure 2, top left) is first distorted by adding Gaussian white
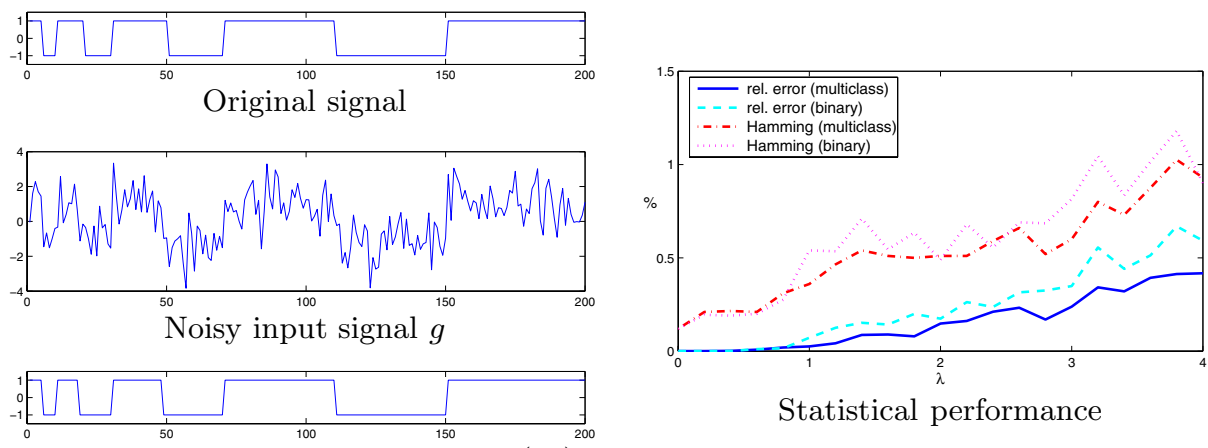

Solution based on SDP relaxation (13)

Fig. 2. One-dimensional restoration. The original signal (top left) is distorted by Gaussian white noise (middle left) and restored based on the SDP relaxation (13). Giving relative errors mostly below $1 \%$, our multiclass SDP relaxation in general performs slightly better in comparison to the binary SDP relaxation presented in 9] (right). 
noise and then restored with the SDP relaxation approach - see Figure 2 (left) for a representative example. The energy $E(x)$ in this case is defined by Potts interaction penalties (5) and assignment costs

$$
C_{i}(a)=\left(u_{a}-g_{i}\right)^{\top}\left(u_{a}-g_{i}\right),
$$

where $g_{i}$ denotes the local measurement at point $i$ and $u_{1}=1, u_{2}=-1$ are the two prototypical class labels. As each signal element is only connected to its two neighbors, the optimal solution $X^{*}$ of (6) can be found easily in this case (for fixed smoothness parameter $\lambda$ ), e.g. by dynamic programming. Comparing $X^{*}$ with the combinatorial solution obtained with the SDP relaxation, we then calculate the relative error of the objective value and the corresponding relative Hamming distance (percentage of misclassified elements).

In order to derive some significant statistics, we perform this experiment for 100 different noisy versions of the original signal, and calculate the corresponding mean errors. The results obtained for a variety of fixed $\lambda$-values are depicted in Figure 2, right. They show that the solutions based on the SDP relaxation approach are remarkably good: the average relative error of the objective value and the average relative Hamming distance both are below 1.5\%, with standard deviations below $0.9 \%$ (objective error) and $1.5 \%$ (Hamming distance), respectively. Note that in this experiment, we do not measure the quality of the restoration, which also depends on picking a suitable value $\lambda$, but the performance of the SDP relaxation approach in relation to the optimal solution.

For comparison, we also performed the same statistical experiment for the $d i$ rect binary SDP relaxation technique presented in [9]. Although the experiments reveal that the objective values $s_{p}^{*}$ of both relaxations coincide (meaning that they are equally tight), a slightly better performance of the multiclass SDP relaxation approach can be observed (see Figure 2, right). This difference indicates that the randomized hyperplane technique used in [9] to obtain a combinatorial solution performs worse than the more sophisticated method used here to find the indicator vectors from the first column $Y_{1}$ of the solution of the multiclass relaxation (13). However, the larger problem size of the multiclass SDP relaxation $(401 \times 401$ compared to $201 \times 201$ for binary relaxation) increases the computational requirements: whereas the solution of the direct binary SDP relaxation is calculated in less than a second, it takes 6-7 seconds to solve the multiclass relaxation (13).

Multiclass Image Labeling. Figure 3 indicates the main characteristics of our SDP relaxation approach for image labeling with a first synthetic example: the restoration of a noisy image originally comprising multiple blocks of different gray-values. For this and all the following two-dimensional experiments, we use a second-order neighborhood structure (horizontally, vertically and diagonally adjacent points are connected), and define separation costs based on Potts interactions penalties (5) with distance weighted values $P_{i j}=\frac{1}{\operatorname{dist}(i, j)} \lambda$. Moreover, assignment costs of type (16) are used, based on suitable prototypes $u_{1}, \ldots, u_{k} \in \mathbb{R}^{m}$ for each class that are fixed in advance. As this type of energy function satisfies the requirements given in [13, we also compare our results 


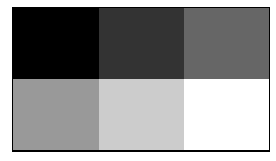

Original

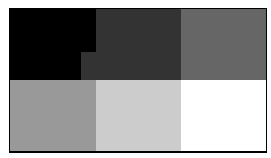

SDP restoration

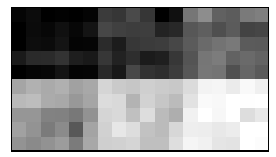

Noisy input

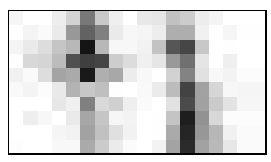

Confidence

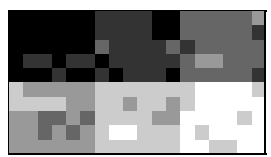

ML classification

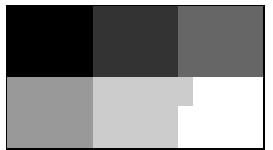

Graph cuts restoration

Fig. 3. Multiclass image labeling result. The original image (top left) of $10 \times$ 18 pixels is degraded by adding Gaussian white noise (top middle). Without spatial relations, ML classification yields a noisy restoration (top right). In contrast, the result of the reconstruction obtained with SDP relaxation (with $\lambda=0.01$ ) is almost perfect: only two pixels are classified incorrectly (bottom left), with high confidence for most pixel labels (bottom middle). This result is comparable to the corresponding restoration obtained with graph cuts (bottom right).

with the corresponding restorations achieved with the expansion move graph cuts algorithm from [5].

The reconstruction obtained with the SDP relaxation method for the small image in Figure 3 is very promising: for this example comprising $k=6$ classes, only two of 180 pixels are labeled incorrectly. Hence, it is of the same quality as the corresponding graph cuts restoration, which also mislabels two pixels. In contrast to that, a simple maximum likelihood (ML) classification (which does not use spatial context) yields a much noisier result (see Figure 3). Interpretation of the solution values $y_{i}$ as probabilities allows us to compute confidence values of the pixel labels by subtracting the two highest probabilities from each other: the results show that only some boundary points receive uncertain labels (dark pixels in Figure 3, bottom middle). Finally, we note that the lower bound $s_{p}^{*}$ on the objective value of (6) computed by the relaxation (13) permits to estimate the performance of the labeling method: comparison with the value $z_{s d p}$ of the final combinatorial solution indicates that the relative error of the result is at most $\frac{z_{s d p}-z^{*}}{z^{*}} \leq \frac{z_{s d p}-s_{p}^{*}}{s_{p}^{*}}=0.064$ for this instance.

Figure 4 depicts more results obtained for different types of image labeling problems. Due to the involved problem sizes (see below), we restrict the algorithm to smaller patches of the original images here. The first row shows the reconstruction of a noisy grayvalue image taken from [5]. Whereas the ML classification is very noisy due to its local behaviour, both the SDP relaxation and graph cuts produce nearly optimal restorations. The example given in the second row demonstrates how the SDP relaxation approach can be applied to natural color images: using the three main colors from the noisy patch as prototypes, a satisfactory classification is obtained (as the original image comprises more than three colors, this cannot be called a 'restoration'). Color differences are calculated in the perceptually uniform $\mathrm{L}^{*} \mathrm{u}^{*} \mathrm{v}^{*}$ space in this case. 

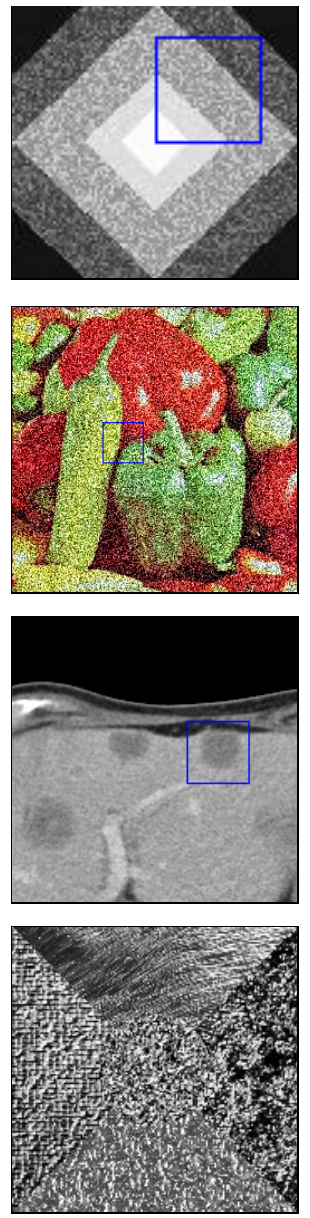

Input

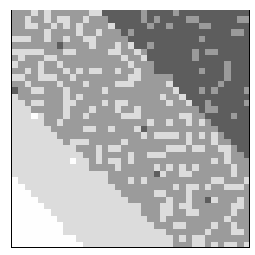

$n=37 \times 37, k=4$

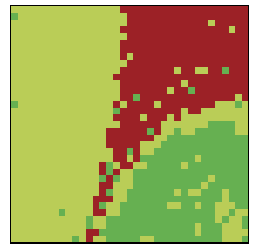

$n=35 \times 35, k=3$

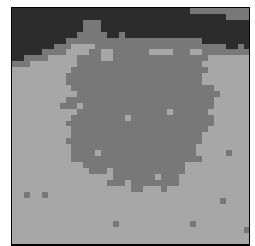

$n=40 \times 40, k=3$

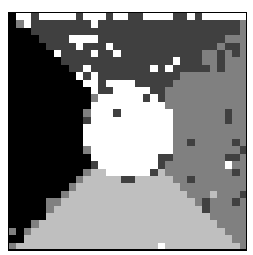

$n=32 \times 32, k=5$

ML classification
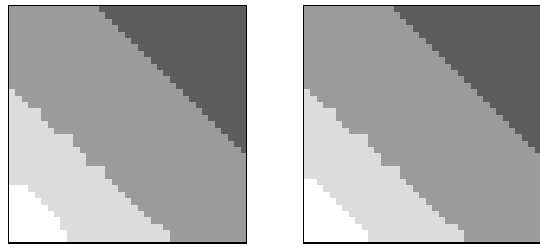

$\lambda=0.01$
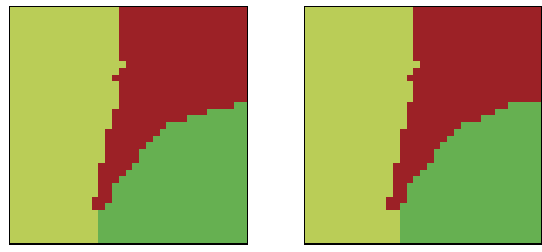

$\lambda=0.02$
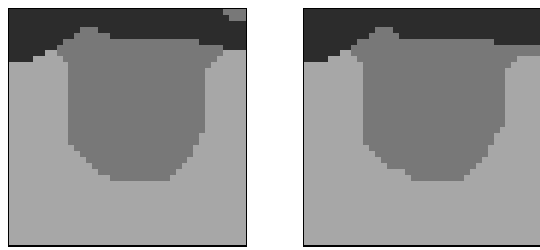

$\lambda=0.02$
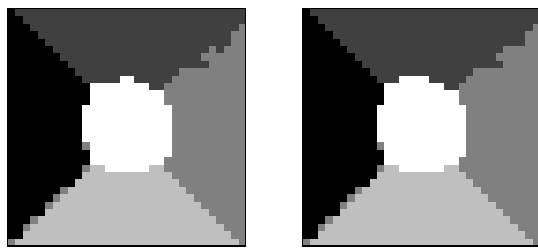

$\lambda=0.05$

SDP labeling Graph cuts labeling

Fig. 4. Multiclass image labeling results. Patches of larger noisy images are restored (rows 1-3) resp. labeled according to texture (row 4) with the SDP relaxation approach. While getting superior results in comparison to a simple maximum likelihood classification, the labelings are of the same quality as those obtained with graph cuts.

The third row of Figure 4 indicates an application of our image labeling method for segmenting tumors in noisy medical images. In this case, prototypes are calculated directly from the image by choosing representative points for each class and averaging the gray values over their neighborhoods. The SDP relaxation then yields a corresponding smooth labeling of the pixels. The last row shows that our SDP relaxation can also successfully be applied for texture classification: dividing the original image into patches of size $16 \times 16$, we compute feature vectors for each patch by averaging the absolute log-Gabor filter convolutions of the corresponding pixels. We then pick representative patches for each class, 
and additionally calculate a diagonal covariance matrix $\Sigma$ from the distribution of filter responses within these patches to obtain more robust assignment costs $C_{i}(a)=\left(u_{a}-g_{i}\right)^{\top} \Sigma^{-1}\left(u_{a}-g_{i}\right)$. Again, the SDP relaxation results in a smooth classification of the same quality as the corresponding graph cuts labeling.

The good performance of the SDP relaxation is also confirmed by the estimates of the relative error obtained from the lower bounds $s_{p}^{*}$, which range between 1\%-4\% for the examples in Figure 4. On the other hand, solving the SDP relaxation is more involved than applying graph cuts: the problem size increases quadratically with $n k$, which results in computation times of up to 4 hours for the examples in Figure 4 in comparison to about one second for finding the corresponding graph cuts solutions. However, these increased computational requirements also make the approach less restrictive, as we will show next.

Image Labeling with Basic Shape Knowledge. In order to demonstrate the generality of our SDP relaxation, we apply it to an image labeling task that in general cannot be handled by other approaches like graph cuts [13. Assuming that some specific information is available on the arrangement of certain label pairs, it is possible to incorporate a basic concept of 'shape' into the labeling problem. For example, it may be known that two labels $a, b$ cannot be neighbored vertically. This information is easily included into the general energy functional (11) by setting $V_{i j}(a, b)$ to a high value whenever $i$ and $j$ are vertical neighbors, and to a small value otherwise. Note that besides preventing the application of methods that rely on a decomposition (3) of the interaction terms [8] (which do not permit label dependent penalties for different neighbor constellations), this might also result in violations of the triangle inequality-type requirements on the interactions necessary e.g. for the expansion move graph cuts algorithm [13.

Figure 5 shows some illustrative examples. To obtain a perfect reconstruction for the image from Figure 3, we simply increase separation costs $V_{i j}(a, b)$ by multiplying them with $\alpha=3$ for vertically neighboring pixels $i, j$ for all three label pairs $a, b$ contained in the top part of the image. In this way, horizontal label changes are preferred over vertical ones, which efficiently prevents the wrong classification of the two pixels in the top left block. The second example is handled similarly, but this time we need to modify interactions differently according to the label constellations: denoting the labels of the left part as $a$ and of the right part as $b_{1}, b_{2}$, respectively, the penalties $V_{i j}\left(a, b_{r}\right)$ are increased for vertical neighbors $i, j$, while $V_{i j}\left(b_{1}, b_{2}\right)$ is increased for horizontal neighbors. Although not perfect, the result clearly demonstrates the influence of these priors.

The last row of Figure 5 indicates how the classification can be restricted to find rectangular shapes: dividing the background into two classes $a_{1}$ and $a_{2}$ neighbored vertically and horizontally to the shape class $b$, respectively, we generally decrease $V_{i j}\left(a_{1}, a_{2}\right)$ to allow pixels to vary from one background class to the other (without changing the assignment costs $C_{i}\left(a_{1}\right)=C_{i}\left(a_{2}\right)$ ), but increase vertical penalties $V_{i j}\left(b, a_{2}\right)$ and $V_{i j}\left(a_{1}, a_{2}\right)$ and horizontal penalties $V_{i j}\left(b, a_{1}\right)$ to model the general label arrangement. The result clearly demonstrates that these modified separation costs successfully restrict the labeling to a rectangular shape, whereas the original interaction values give an inferior reconstruction. 

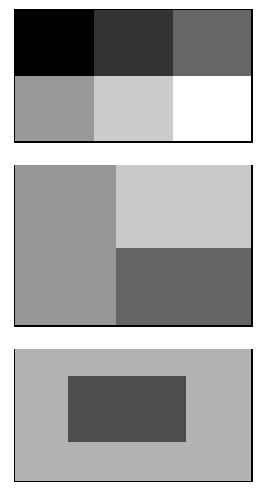

Original
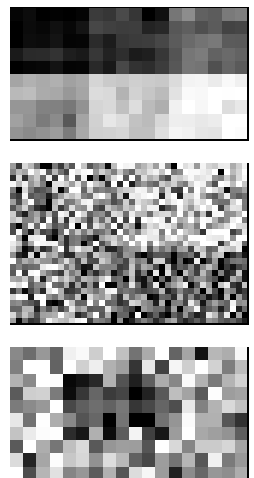

Noisy input
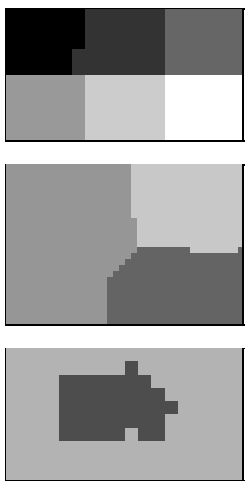

SDP labeling
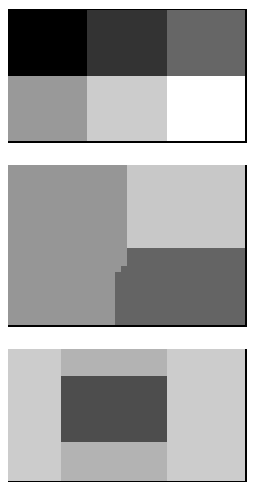

SDP label. with prior

Fig. 5. Image labeling with basic 'shape' concept. Including simple information about preferred label arrangements results in better reconstructions of heavily noisy images. The slightly varying background color in the bottom right image visualizes the artificially enforced splitting into two different background classes.

\section{Conclusion}

We have presented a method for multiclass image labeling that is able to find approximate solutions of high quality for a very general class of combinatorial optimization problems. Applying the mathematically fundamental concept of Lagrangian relaxation, we obtain a semidefinite program which due to its convexity can be solved in polynomial time without having to optimize any tuning parameters. In fact, besides defining the classes, only the parameter $\lambda$ controlling the smoothness of the result needs to be adjusted. In comparison to other approaches like graph cuts, our SDP relaxation method is not restricted to special types of the energy function, and additionally gives a lower bound on the optimal solution value that can be used to estimate the relative error of the result for each problem instance.

The generality of our method enables us to incorporate a basic concept of shape into the labeling process. It will be interesting to further investigate this idea to see whether more complex shape information can be included.

However, this generality of the multiclass SDP relaxation approach has its price: since the problem size increases quadratically with the product of the number of image elements and the number of classes, the application of this method is (yet) restricted to small optimization problems consisting of only few different classes. In order to remedy this drawback one could perform labeling successively on different scales: Extracting larger image objects, a corresponding coarse scale classification is computed in a first step. Afterwards, the labeling can be refined by applying fine scale classification to those objects that received low confidence values. In this way, instead of having to compute the solution for one large problem instance, a sequence of smaller problems that can be solved more efficiently needs to be considered. 
Finally, we note that it is often possible to tighten Lagrangian relaxations by incorporating additional constraints that are redundant for the original problem (cf. [10]). Future work will show whether this is useful in our case to find better approximative solutions.

\section{References}

1. Winkler, G.: Image Analysis, Random Fields and Dynamic Monte Carlo Methods. Volume 27 of Appl. of Mathematics. Springer (1995)

2. Geman, S., Geman, D.: Stochastic relaxation, Gibbs distributions, and the Bayesian restoration of images. IEEE Trans. PAMI 6 (1984) 721-741

3. Besag, J.: On the statistical analysis of dirty pictures. Journal of the Royal Statistical Society, Series B 48 (1986) 259-302

4. Li, S.Z.: Markov Random Field Modeling in Image Analysis. Springer (2001)

5. Boykov, Y., Veksler, O., Zabih, R.: Fast approximate energy minimization via graph cuts. IEEE Trans. PAMI 23 (2001) 1222-1239

6. Blake, A., Zisserman, A.: Visual Reconstruction. MIT Press (1987)

7. Hofmann, T., Buhmann, J.: Pairwise data clustering by deterministic annealing. IEEE Trans. PAMI 19 (1997) 1-14

8. Kleinberg, J., Tardos, E.: Approximation algorithms for classification problems with pairwise relationships: Metric labeling and Markov random fields. Journal of the ACM 49 (2002) 616-639

9. Keuchel, J., Schnörr, C., Schellewald, C., Cremers, D.: Binary partitioning, perceptual grouping, and restoration with semidefinite programming. IEEE Trans. PAMI 25 (2003) 1364-1379

10. Zhao, Q., Karisch, S.E., Rendl, F., Wolkowicz, H.: Semidefinite programming relaxations for the quadratic assignment problem. J. Combinat. Optim. 2 (1998) 71-109

11. Wolkowicz, H., Anjos, M.F.: Semidefinite programming for discrete optimization and matrix completion problems. Discr. Appl. Math. 123 (2002) 513-577

12. Laurent, M., Rendl, F.: Semidefinite programming and integer programming. In Aardal, K., Nemhauser, G.L., Weismantel, R., eds.: Discrete Optimization. Volume 12 of Handbooks in Op. Res. and Man. Sci. Elsevier (2005)

13. Kolmogorov, V., Zabih, R.: What energy functions can be minimized via graph cuts? IEEE Trans. PAMI 26 (2004) 147-159

14. Rother, C., Kumar, S., Kolmogorov, V., Blake, A.: Digital tapestry. In: Proceedings CVPR (1). (2005) 589-596

15. Raj, A., Zabih, R.: A graph cut algorithm for generalized image deconvolution. In: Proc. 10th Int. Conf. Computer Vision (ICCV). (2005) 1048-1054

16. Wolkowicz, H., Saigal, R., Vandenberghe, L., eds.: Handbook of Semidefinite Programming. Volume 27 of International Series in Operations Research \& Management Science. Kluwer Acad. Publ., Boston (2000)

17. Çela, E.: The Quadratic Assignment Problem: Theory and Algorithms. Kluwer Acad. Publishers, Dordrecht (1998)

18. Brixius, N., Anstreicher, K.: Solving quadratic assignment problems using convex quadratic programming relaxations. Optim. Methods Software 16 (2001) 49-68

19. Alizadeh, F.: Interior point methods in semidefinite programming with applications to combinatorial optimization. SIAM J. Optimization 5 (1995) 13-51

20. Kočvara, M., Stingl, M.: PENNON - a code for convex nonlinear and semidefinite programming. Optimization Methods and Software 18 (2003) 317-333 